\title{
Triply-charmed dibaryon states or two-baryon scattering states from QCD sum rules
}

\author{
Zhi-Gang Wang ( ${ }^{*}$ \\ Department of Physics, North China Electric Power University, \\ Baoding 071003, People's Republic of China
}

(Received 16 December 2019; accepted 29 July 2020; published 7 August 2020)

\begin{abstract}
In this article, we construct the color-singlet-color-singlet type currents to study the scalar and axialvector $\Xi_{c c} \Sigma_{c}$ dibaryon states with QCD sum rules in details by taking into account both the dibaryon states and two-baryon scattering sates at the hadron side, and examine the existence of the $\Xi_{c c} \Sigma_{c}$ dibaryon states. Our calculations indicate that the two-baryon scattering states cannot saturate the QCD sum rules, it is necessary to introduce the dibaryon states, the color-singlet-color-singlet type currents couple potentially to the molecular states, not to the two-particle scattering states, the molecular states begin to receive contributions at the order $\mathcal{O}\left(\alpha_{s}^{0}\right)$, not at the order $\mathcal{O}\left(\alpha_{s}^{2}\right)$.
\end{abstract}

DOI: $10.1103 /$ PhysRevD.102.034008

\section{INTRODUCTION}

A dibaryon state denotes an object with baryon number 2 , the oldest known dibaryon state is the deuteron, which is a very loosely bound state of two baryons, a proton and a neutron, and is made of six light valence quarks. In 2014, the WASA-at-COSY collaboration established the narrow resonance structure $d^{*}(2380)$ with $I\left(J^{P}\right)=0\left(3^{+}\right)$as a genuine $s$-channel resonance using partial-wave analysis, and given the first clear-cut experimental evidence for the existence of a true dibaryon resonance [1], the $d^{*}(2380)$ was firstly observed in the double-pionic fusion to the deuteron [2]. The $d^{*}(2380)$ may be a $\Delta \Delta$ dibaryon state or a six-quark state, for more theoretical and experimental works on the light flavor dibaryon states, one can consult the comprehensive review [3].

On the other hand, many charmoniumlike and bottomoniumlike states were observed after the discovery of the $X(3872)$ by the Belle collaboration in 2003 [4]. It is difficult to accommodate those exotic $X, Y$, and $Z$ states in the conventional meson spectrum, especially, the charged charmoniumlike states are good candidates for the multiquark states, tetraquark states, or molecular states [5].

The observation of the $d^{*}(2380)$ triggers much theoretical interest on possible existence of the molecular states made of two heavy baryons. As the large masses of the heavy baryons reduce the kinetic energy of the two-baryon

\footnotetext{
*zgwang@aliyun.com.
}

Published by the American Physical Society under the terms of the Creative Commons Attribution 4.0 International license. Further distribution of this work must maintain attribution to the author(s) and the published article's title, journal citation, and DOI. Funded by SCOAP. systems, which makes it easier to form bound states. In 2015 , the LHCb collaboration observed two pentaquark candidates $P_{c}(4380)$ and $P_{c}(4450)$ in the $J / \psi p$ mass spectrum [6]. In 2019, the LHCb collaboration observed a narrow pentaquark candidate $P_{c}(4312)$ and confirmed the $P_{c}(4450)$ pentaquark structure, which consists of two narrow overlapping peaks $P_{c}(4440)$ and $P_{c}(4457)$ [7]. The $P_{c}$ (4312), $P_{c}(4380), P_{c}(4440)$, and $P_{c}(4457)$ lie near the $\bar{D} \Sigma_{c}, \bar{D} \Sigma_{c}^{*}, \bar{D}^{*} \Sigma_{c}$, and $\bar{D}^{*} \Sigma_{c}^{*}$ thresholds respectively, which leads to the conjecture that they are meson-baryon type molecular states. We can learn something about the $\bar{D} \Sigma_{c}, \bar{D} \Sigma_{c}^{*}, \bar{D}^{*} \Sigma_{c}$, and $\bar{D}^{*} \Sigma_{c}^{*}$ pentaquark molecular states from the $\Xi_{c c} \Sigma_{c}, \Xi_{c c} \Sigma_{c}^{*}, \Xi_{c c}^{*} \Sigma_{c}$, and $\Xi_{c c}^{*} \Sigma_{c}^{*}$ dibaryon states (or vice versa), which are connected with each other by heavy antiquark-diquark symmetry, if we assume the lightquark structures are almost identical [8]. In Ref. [9], the molecular states consist of a doubly charmed baryon and an $\mathrm{S}$-wave charmed baryon are investigated in the one-bosonexchange model.

Exploring the hadron-hadron interactions plays an important role in understanding the meson-meson type, meson-baryon type, baryon-antibaryon type, baryonbaryon type molecular states. It is essential to make a detailed theoretical investigation of those molecular states to encourage or stimulate new experiments to search for some evidences. Theoretically, we can study the molecular states at the hadron level [8-10] or at the quark level $[11,12]$.

In Ref. [11], Junnarkar and Mathur study the $\Sigma_{c} \Xi_{c c}$, $\Omega_{c} \Omega_{c c}, \Sigma_{b} \Xi_{b b}, \Omega_{b} \Omega_{b b}$, and $\Omega_{c c b} \Omega_{c b b}$ dibaryon states with $J^{P}=1^{+}$via the lattice $\mathrm{QCD}$, and unambiguously find that the ground state masses of the dibaryon states $\Omega_{c} \Omega_{c c}$, $\Omega_{b} \Omega_{b b}$, and $\Omega_{c c b} \Omega_{c b b}$ are below their respective twobaryon thresholds, but cannot obtain definitive conclusion 
about the existence of the $\Sigma_{c} \Xi_{c c}$ and $\Sigma_{b} \Xi_{b b}$ dibaryon states due to large systematic errors. The doubly or triply heavy baryon states have not been observed experimentally yet except for the $\Xi_{c c}$ [13]. So it is interesting to study the $\Sigma_{c} \Xi_{c c}$ dibaryon states with the QCD sum rules, as we have experimental input to assess the bound energies. In Ref. [14], the $H$-dibaryon or $\Lambda \Lambda$ dibaryon state is studied with the QCD sum rules. In Ref. [15], the $d^{*}(2380)$ is assigned as a $\Delta \Delta$ dibaryon state and studied with the QCD sum rules.

The QCD sum rules approach is a powerful nonperturbative tool in studying the ground state tetraquark states, tetraquark molecular states, pentaquark states, pentaquark molecular states, and has given many successful descriptions of the hadronic parameters, such as the masses and decay widths [16-23]. However, a different voice arises, Lucha, Melikhov, and Sazdjian assert that the QCD sum rules are misused to study the tetraquark or molecule masses, all the four-quark currents can be rearranged into the color-singlet-color-singlet type currents, the contributions at the order $\mathcal{O}\left(\alpha_{s}^{k}\right)$ with $k \leq 1$ in the operator product expansion, which are factorizable in the color space, are exactly canceled out by the two-meson scattering states at the hadron side, the tetraquark molecular states begin to receive contributions at the order $\mathcal{O}\left(\alpha_{s}^{2}\right)$ [24,25]. The tetraquark molecular states are two-meson bound states or resonant states in contrast to the two-meson scattering states. The dibaryon states consist of two color-singlet objects, they are an another type molecular states. In this article, we will examine the applicability of the QCD sum rules to study the two-baryon type molecular states.

In this article, we study the scalar and axialvector $\Xi_{c c} \Sigma_{c}$ dibaryon states with QCD sum rules in details. We take into account both the dibaryon states and twobaryon scattering sates at the hadron side, and examine whether or not the QCD sum rules support the existences of dibaryon states.

The article is arranged as follows: we obtain the QCD sum rules for the masses and pole residues of the triplycharmed dibaryon states and examine the applicability of the QCD sum rules in Sec. II; in Sec. III, we present the numerical results and discussions; and Sec. IV is reserved for our conclusion.

\section{QCD SUM RULES FOR THE DIBARYON STATES}

In the following, we write down the two-point correlation functions $\Pi_{S}(p)$ and $\Pi_{\mu \nu}(p)$ in the QCD sum rules,

$$
\begin{aligned}
\Pi_{S}(p) & =i \int d^{4} x e^{i p \cdot x}\left\langle 0\left|T\left\{J(x) J^{\dagger}(0)\right\}\right| 0\right\rangle, \\
\Pi_{\mu \nu}(p) & =i \int d^{4} x e^{i p \cdot x}\left\langle 0\left|T\left\{J_{\mu}(x) J_{\nu}^{\dagger}(0)\right\}\right| 0\right\rangle,
\end{aligned}
$$

where

$$
\begin{aligned}
J(x) & =J_{c}^{T}(x) C \gamma_{5} J_{c c}(x), \\
J_{\mu}(x) & =J_{c}^{T}(x) C \gamma_{\mu} J_{c c}(x), \\
J_{c}(x) & =\varepsilon^{i j k} q_{i}^{T}(x) C \gamma_{\alpha} q_{j}(x) \gamma^{\alpha} \gamma_{5} c_{k}(x), \\
J_{c c}(x) & =\varepsilon^{i j k} c_{i}^{T}(x) C \gamma_{\alpha} c_{j}(x) \gamma^{\alpha} \gamma_{5} q_{k}(x),
\end{aligned}
$$

the $i, j$, and $k$ are color indexes, the $C$ is the charge conjugation matrix. The currents $J(x)$ and $J_{\mu}(x)$ have two color-neutral clusters and have the property under parity transformation,

$$
\begin{aligned}
\hat{P} J(x) \hat{P}^{-1} & =+J(\tilde{x}), \\
\hat{P} J_{\mu}(x) \hat{P}^{-1} & =-J^{\mu}(\tilde{x}),
\end{aligned}
$$

where the coordinates $x^{\mu}=(t, \vec{x})$ and $\tilde{x}^{\mu}=(t,-\vec{x})$.

We construct the Ioffe's currents $J_{c}(x)$ and $J_{c c}(x)$ according to the Fermi-Dirac statistics and the attractive interactions originate from the one-gluon exchange. The currents $J_{c}(x)$ and $J_{c c}(x)$ have the $J^{P}=\frac{1}{2}^{+}$and have the constituent quarks or valence quarks $q q c$ and $c c q$, respectively, just like the baryon states $\Sigma_{c}$ and $\Xi_{c c}$. The quantum field theory does not forbid the current-baryon couplings,

$$
\begin{aligned}
\left\langle 0\left|J_{c}(0)\right| \Sigma_{c}(p)\right\rangle & =\lambda_{\Sigma} U_{\Sigma}(p), \\
\left\langle 0\left|J_{c c}(0)\right| \Xi_{c c}(p)\right\rangle & =\lambda_{\Xi} U_{\Xi}(p),
\end{aligned}
$$

the $U_{\Sigma}(p)$ and $U_{\Xi}(p)$ are Dirac spinors. The values of the coupling constants or pole residues $\lambda_{\Sigma}$ and $\lambda_{\Xi}$ are not experimentally measurable quantities, we have to calculate those values with the QCD sum rules or lattice QCD.

The $\Sigma_{c} \Xi_{c c}$ systems maybe form the $\Sigma_{c} \Xi_{c c}$ dibaryon states, or maybe not form the $\Sigma_{c} \Xi_{c c}$ dibaryon states (in other words, they are just the $\Sigma_{c} \Xi_{c c}$ two-baryon scattering states). If they form the $\Sigma_{c} \Xi_{c c}$ dibaryon states, the quantum field theory does not forbid the current-dibaryon couplings, the currents $J(x)$ and $J_{\mu}(x)$ couple potentially to the scalar and axialvector dibaryon states, respectively, or to the twobaryon scattering states with the spin-parity $J^{P}=0^{+}$and $1^{+}$, respectively,

$$
\begin{aligned}
\left\langle 0|J(0)| D_{S}(p)\right\rangle & =\lambda_{S}, \\
\left\langle 0\left|J_{\mu}(0)\right| D_{A}(p)\right\rangle & =\lambda_{A} \varepsilon_{\mu}, \\
\left\langle 0|J(0)| \Sigma_{c}(q) \Xi_{c c}(p-q)\right\rangle & =\lambda_{\Sigma} \lambda_{\Xi} U_{\Sigma}^{T}(q) C \gamma_{5} U_{\Xi}(p-q), \\
\left\langle 0\left|J_{\mu}(0)\right| \Sigma_{c}(q) \Xi_{c c}(p-q)\right\rangle & =\lambda_{\Sigma} \lambda_{\Xi} U_{\Sigma}^{T}(q) C \gamma_{\mu} U_{\Xi}(p-q),
\end{aligned}
$$

we use the $D$ to denote the dibaryon states.

At the hadron side of the correlation functions $\Pi_{S}(p)$ and $\Pi_{\mu \nu}(p)$, we isolate the contributions of both the lowest dibaryon states and two-baryon scattering states,

$$
\Pi_{\mu \nu}(p)=\Pi_{A}(p)\left(-g_{\mu \nu}+\frac{p_{\mu} p_{\nu}}{p^{2}}\right)+\cdots,
$$




$$
\begin{aligned}
& \Pi_{S}(p)=\frac{\lambda_{S}^{2}}{M_{S}^{2}-p^{2}}+\int_{\Delta^{2}}^{s_{0}} d s \frac{1}{s-p^{2}} \rho_{H, S}(s)+\cdots, \\
& \Pi_{A}(p)=\frac{\lambda_{A}^{2}}{M_{A}^{2}-p^{2}}+\int_{\Delta^{2}}^{s_{0}} d s \frac{1}{s-p^{2}} \rho_{H, A}(s)+\cdots,
\end{aligned}
$$

where

$$
\begin{aligned}
\rho_{H, S} & =\frac{\lambda_{\Sigma}^{2} \lambda_{\Xi}^{2}}{8 \pi^{2}} \frac{\sqrt{\lambda\left(s, m_{\Sigma}^{2}, m_{\Xi}^{2}\right)}}{s}\left[s-\left(m_{\Xi}-m_{\Sigma}\right)^{2}\right], \\
\rho_{H, A} & =\frac{\lambda_{\Sigma}^{2} \lambda_{\Xi}^{2}}{8 \pi^{2}} \frac{\sqrt{\lambda\left(s, m_{\Sigma}^{2}, m_{\Xi}^{2}\right)}}{s}\left[s-\left(m_{\Xi}-m_{\Sigma}\right)^{2}-\frac{\lambda\left(s, m_{\Sigma}^{2}, m_{\Xi}^{2}\right)}{3 s}\right],
\end{aligned}
$$

where $\Delta^{2}=\left(m_{\Sigma}+m_{\Xi}\right)^{2}, \lambda(a, b, c)=a^{2}+b^{2}+c^{2}-2 a b-2 b c-2 a c$, the $s_{0}$ are the continuum threshold parameters.

In the QCD sum rules, we carry out the operator product expansion at the deep Euclidean region $P^{2}=-p^{2} \rightarrow \infty$, which corresponds to the small spatial distance and time interval $x_{0} \sim \vec{x} \sim \frac{1}{\sqrt{P^{2}}}$, and $x^{2} \sim \frac{1}{P^{2}}$, it is questionable to apply the Landau equation to study the Feynman diagrams [26]. At the QCD side of the correlation functions $\Pi_{S}(p)$ and $\Pi_{\mu \nu}(p)$, we contract the $q$ and $c$ quark fields with Wick theorem and obtain the results,

$$
\begin{aligned}
& \Pi_{S}(p)=-i \varepsilon^{i j k} \varepsilon^{l m n} \varepsilon^{i^{\prime} j^{\prime} k^{\prime}} \varepsilon^{l^{\prime} m^{\prime} n^{\prime}} \int d^{4} x e^{i p \cdot x}\left\{4 \operatorname{Tr}\left[\gamma_{\alpha} S^{j j^{\prime}}(x) \gamma_{\alpha^{\prime}} C S^{i i^{\prime} T}(x) C\right] \operatorname{Tr}\left[\gamma_{\beta} S_{c}^{m m^{\prime}}(x) \gamma_{\beta^{\prime}} C S_{c}^{l l^{\prime} T}(x) C\right]\right. \\
& \times \operatorname{Tr}\left[\gamma_{5} \gamma^{\alpha} \gamma_{5} \gamma^{\beta} \gamma_{5} S^{n n^{\prime}}(x) \gamma_{5} \gamma^{\beta^{\prime}} \gamma_{5} \gamma^{\alpha^{\prime}} \gamma_{5} C S_{c}^{k k^{\prime} T}(x) C\right] \\
& -8 \operatorname{Tr}\left[\gamma_{\alpha} S^{j j^{\prime}}(x) \gamma_{\alpha^{\prime}} C S^{i i^{\prime} T}(x) C\right] \operatorname{Tr}\left[\gamma_{5} \gamma^{\alpha} \gamma_{5} \gamma^{\beta} \gamma_{5} S^{n n^{\prime}}(x) \gamma_{5} \gamma^{\beta^{\prime}} \gamma_{5} \gamma^{\alpha^{\prime}} \gamma_{5} C S_{c}^{m k^{\prime} T}(x) C \gamma_{\beta} S_{c}^{l l^{\prime}}(x) \gamma_{\beta^{\prime}} C S_{c}^{k m^{\prime} T}(x) C\right] \\
& -8 \operatorname{Tr}\left[\gamma_{5} \gamma^{\alpha} \gamma_{5} \gamma^{\beta} \gamma_{5} S^{n j^{\prime}}(x) \gamma_{\alpha^{\prime}} C S^{i i^{\prime} T}(x) C \gamma_{\alpha} S^{j n^{\prime}}(x) \gamma_{5} \gamma^{\beta^{\prime}} \gamma_{5} \gamma^{\alpha^{\prime}} \gamma_{5} C S_{c}^{k k^{\prime} T}(x) C\right] \operatorname{Tr}\left[\gamma_{\beta} S_{c}^{m m^{\prime}}(x) \gamma_{\beta^{\prime}} C S_{c}^{l l^{\prime} T}(x) C\right] \\
& \left.+16 \operatorname{Tr}\left[\gamma_{5} \gamma^{\alpha} \gamma_{5} \gamma^{\beta} \gamma_{5} S^{n j^{\prime}}(x) \gamma_{\alpha^{\prime}} C S^{i i^{\prime} T}(x) C \gamma_{\alpha} S^{j n^{\prime}}(x) \gamma_{5} \gamma^{\beta^{\prime}} \gamma_{5} \gamma^{\alpha^{\prime}} \gamma_{5} C S_{c}^{m k^{\prime} T}(x) C \gamma_{\beta} S_{c}^{l l^{\prime}}(x) \gamma_{\beta^{\prime}} C S_{c}^{k m^{\prime} T}(x) C\right]\right\} \text {, } \\
& \Pi_{\mu \nu}(p)=i \varepsilon^{i j k} \varepsilon^{l m n} \varepsilon^{i^{\prime} j^{\prime} k^{\prime}} \varepsilon^{l^{\prime} m^{\prime} n^{\prime}} \int d^{4} x e^{i p \cdot x}\left\{4 \operatorname{Tr}\left[\gamma_{\alpha} S^{j j^{\prime}}(x) \gamma_{\alpha^{\prime}} C S^{i i^{\prime} T}(x) C\right] \operatorname{Tr}\left[\gamma_{\beta} S_{c}^{m m^{\prime}}(x) \gamma_{\beta^{\prime}} C S_{c}^{l l^{\prime} T}(x) C\right]\right. \\
& \times \operatorname{Tr}\left[\gamma_{5} \gamma^{\alpha} \gamma_{\mu} \gamma^{\beta} \gamma_{5} S^{n n^{\prime}}(x) \gamma_{5} \gamma^{\beta^{\prime}} \gamma_{\nu} \gamma^{\alpha^{\prime}} \gamma_{5} C S_{c}^{k k^{\prime} T}(x) C\right] \\
& -8 \operatorname{Tr}\left[\gamma_{\alpha} S^{j j^{\prime}}(x) \gamma_{\alpha^{\prime}} C S^{i i^{\prime} T}(x) C\right] \operatorname{Tr}\left[\gamma_{5} \gamma^{\alpha} \gamma_{\mu} \gamma^{\beta} \gamma_{5} S^{n n^{\prime}}(x) \gamma_{5} \gamma^{\beta^{\prime}} \gamma_{\nu} \gamma^{\alpha^{\prime}} \gamma_{5} C S_{c}^{m k^{\prime} T}(x) C \gamma_{\beta} S_{c}^{l l^{\prime}}(x) \gamma_{\beta^{\prime}} C S_{c}^{k m^{\prime} T}(x) C\right] \\
& -8 \operatorname{Tr}\left[\gamma_{5} \gamma^{\alpha} \gamma_{\mu} \gamma^{\beta} \gamma_{5} S^{n j^{\prime}}(x) \gamma_{\alpha^{\prime}} C S^{i i^{\prime} T}(x) C \gamma_{\alpha} S^{j n^{\prime}}(x) \gamma_{5} \gamma^{\beta^{\prime}} \gamma_{\nu} \gamma^{\alpha^{\prime}} \gamma_{5} C S_{c}^{k k^{\prime} T}(x) C\right] \operatorname{Tr}\left[\gamma_{\beta} S_{c}^{m m^{\prime}}(x) \gamma_{\beta^{\prime}} C S_{c}^{l l^{\prime} T}(x) C\right] \\
& \left.+16 \operatorname{Tr}\left[\gamma_{5} \gamma^{\alpha} \gamma_{\mu} \gamma^{\beta} \gamma_{5} S^{n j^{\prime}}(x) \gamma_{\alpha^{\prime}} C S^{i i^{\prime} T}(x) C \gamma_{\alpha} S^{j n^{\prime}}(x) \gamma_{5} \gamma^{\beta^{\prime}} \gamma_{\nu} \gamma^{\alpha^{\prime}} \gamma_{5} C S_{c}^{m k^{\prime} T}(x) C \gamma_{\beta} S_{c}^{l l^{\prime}}(x) \gamma_{\beta^{\prime}} C S_{c}^{k m^{\prime} T}(x) C\right]\right\} \text {, }
\end{aligned}
$$

where

$$
\begin{gathered}
S_{i j}(x)=\frac{i \delta_{i j} \not x}{2 \pi^{2} x^{4}}-\frac{\delta_{i j}\langle\bar{q} q\rangle}{12}-\frac{\delta_{i j} x^{2}\left\langle\bar{q} g_{s} \sigma G q\right\rangle}{192}-\frac{i g_{s} G_{\alpha \beta}^{a} t_{i j}^{a}\left(\not x \sigma^{\alpha \beta}+\sigma^{\alpha \beta} \not x\right)}{32 \pi^{2} x^{2}} \\
-\frac{\delta_{i j} x^{4}\langle\bar{q} q\rangle\left\langle g_{s}^{2} G G\right\rangle}{27648}-\frac{1}{8}\left\langle\bar{q}_{j} \sigma^{\mu \nu} q_{i}\right\rangle \sigma_{\mu \nu}+\cdots, \\
S_{c}^{i j}(x)=\frac{i}{(2 \pi)^{4}} \int d^{4} k e^{-i k \cdot x}\left\{\frac{\delta_{i j}}{\not k-m_{c}}-\frac{g_{s} G_{\alpha \beta}^{n} t_{i j}^{n}}{4} \frac{\sigma^{\alpha \beta}\left(\not k+m_{c}\right)+\left(\not k+m_{c}\right) \sigma^{\alpha \beta}}{\left(k^{2}-m_{c}^{2}\right)^{2}}\right. \\
\left.-\frac{g_{s}^{2}\left(t^{a} t^{b}\right)_{i j} G_{\alpha \beta}^{a} G_{\mu \nu}^{b}\left(f^{\alpha \beta \mu \nu}+f^{\alpha \mu \beta \nu}+f^{\alpha \mu \nu \beta}\right)}{4\left(k^{2}-m_{c}^{2}\right)^{5}}+\cdots\right\}, \\
f^{\alpha \beta \mu \nu}=\left(\not k+m_{c}\right) \gamma^{\alpha}\left(\not k+m_{c}\right) \gamma^{\beta}\left(\not k+m_{c}\right) \gamma^{\mu}\left(\not k+m_{c}\right) \gamma^{\nu}\left(\not k+m_{c}\right),
\end{gathered}
$$

and $t^{n}=\frac{\lambda^{n}}{2}$, the $\lambda^{n}$ is the Gell-Mann matrix [17,27,28]. In the full light quark propagator, see Eq. (11), we add the term $\left\langle\bar{q}_{j} \sigma_{\mu \nu} q_{i}\right\rangle$ obtained from Fierz rearrangement of the $\left\langle q_{i} \bar{q}_{j}\right\rangle$ to absorb the gluons emitted from other light quark lines and heavy quark lines to extract the mixed condensate $\left\langle\bar{q} g_{s} \sigma G q\right\rangle$ [17]. 

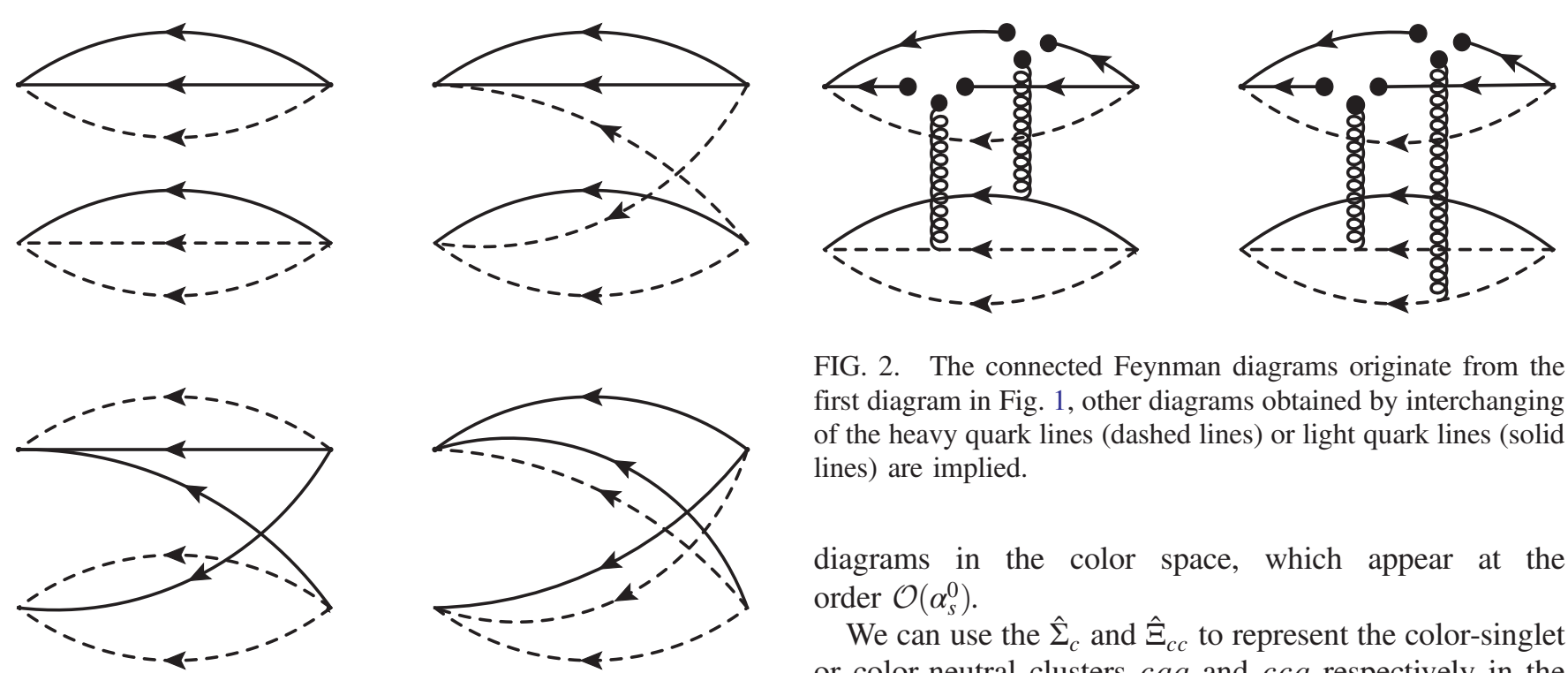

FIG. 2. The connected Feynman diagrams originate from the first diagram in Fig. 1, other diagrams obtained by interchanging of the heavy quark lines (dashed lines) or light quark lines (solid lines) are implied.

FIG. 1. The Feynman diagrams for the lowest order contributions, where the solid lines and dashed lines represent the light quarks and heavy quarks, respectively.

In Fig. 1, we draw the lowest order Feynman diagrams, which correspond to the perturbative contributions in Eqs. (9) and (10), in other words, the free-quark contributions in the full quark propagators in Eqs. (11) and (12). The first diagram in Fig. 1 is factorizable or disconnected in the color space, the other diagrams are nonfactorizable or connected in the color space. The (non)factorizable properties in the color space are independent of the (non) factorizable properties in the momentum space. In fact, all the Feynman diagrams are nonfactorizable in the momentum space. Thereafter, we will neglect the phrase "in the color space" for simplicity when discuss the (non)factorizable properties in most cases.

If we substitute the light-quark lines and heavy-quark lines in Fig. 1 with other terms in the full light-quark and heavy-quark propagators in Eqs. (11) and (12), we can obtain all the relevant Feynman diagrams. From the first diagram in Fig. 1, we can obtain both connected and disconnected Feynman diagrams, the connected contributions appear due to the quark-gluon operators $\bar{q} g_{s} G q \bar{q} g_{s} G q$, which are of the order $\mathcal{O}\left(\alpha_{s}^{1}\right)$ and come from the Feynman diagrams shown in Fig. 2. From the quark-gluon operators $\bar{q} g_{s} G q \bar{q} g_{s} G q$, we can obtain the vacuum condensate $\left\langle\bar{q} g_{s} \sigma G q\right\rangle^{2}$, the $g_{s}^{2}=4 \pi \alpha_{s}$ is absorbed into the vacuum condensate, so the diagrams in Fig. 2 can be counted as of the order $\mathcal{O}\left(\alpha_{s}^{0}\right)$. Those contributions should be taken into account as the QCD sum rules is a nonperturbative method, the connected (or nonfactorizable) Feynman diagrams appear at the order $\mathcal{O}\left(\alpha_{s}^{0}\right)$ or $\mathcal{O}\left(\alpha_{s}^{1}\right)$, not at the order $\mathcal{O}\left(\alpha_{s}^{2}\right)$ asserted by Lucha, Melikhov, and Sazdjian in Refs. [24,25]. While from other diagrams in Fig. 1, we can obtain only connected (or nonfactorizable) Feynman

diagrams in the color space, which appear at the order $\mathcal{O}\left(\alpha_{s}^{0}\right)$.

We can use the $\hat{\Sigma}_{c}$ and $\hat{\Xi}_{c c}$ to represent the color-singlet or color-neutral clusters $c q q$ and $c c q$ respectively in the currents. From those Feynman diagrams, we can observe that the initial color-neutral clusters $\hat{\Sigma}_{c}$ and $\hat{\Xi}_{c c}$ evolve to the final color-neutral clusters $\hat{\Sigma}_{c}$ and $\hat{\Xi}_{c c}$ with (without) interchanging colored objects, such as quarks and gluons, in the nonfactorizable (factorizable) Feynman diagrams. The $\Sigma_{c}\left(\Xi_{c c}\right)$ and $\hat{\Sigma}_{c}\left(\hat{\Xi}_{c c}\right)$ have the same quantum numbers $J^{P}=\frac{1}{2}{ }^{+}$, the quantum field theory allows nonvanishing couplings between the $\Sigma_{c}\left(\Xi_{c c}\right)$ and $\hat{\Sigma}_{c}\left(\hat{\Xi}_{c c}\right)$, irrespective of whether the $\Sigma_{c}$ and $\Xi_{c c}$ are the two-baryon scattering states or the $\Sigma_{c} \Xi_{c c}$ components in the dibaryon states. We cannot obtain other information about the hadrons beyond that from the Feynman diagrams directly, because we carry out the operator product expansion in the quantum field theory, there are both perturbative and nonperturbative contributions, the vacuum condensates are highly nonperturbative quantities and embody the net collective effects.

In the similar systems, the four-quark systems, Lucha, Melikhov, and Sazdjian assert that the contributions at the order $\mathcal{O}\left(\alpha_{s}^{k}\right)$ with $k \leq 1$ in the operator product expansion, which are factorizable (or disconnected) in the color space, are exactly canceled out by the two-meson scattering states at the hadron side, the connected (or nonfactorizable) Feynman diagrams appear at the order $\mathcal{O}\left(\alpha_{s}^{2}\right)$, if have a Landau singularity, begin to make contributions to the tetraquark state or tetraquark molecular state $[24,25]$.

Without asserting that the factorizable Feynman diagrams in the color space only make contributions to the two-particle scattering states, the Landau equation is irrelevant in selecting the Feynman diagrams, because the factorizable Feynman diagrams in the color space can also have Landau singularities. In fact, the quarks and gluons are confined objects, they cannot be put on the mass-shell, it is questionable to say that the Landau equation is applicably in the nonperturbative QCD calculations involving bound states [26]. Furthermore, even the Landau singularities appear at the order $\mathcal{O}\left(\alpha_{s}^{2}\right)$ according to the assertion of Lucha, Melikhov, and Sazdjian, we cannot 
obtain the conclusion that those Feynman diagrams make contributions to a tetraquark state or tetraquark molecular state, as the Landau singularity only indicates that there exists an intermediate state consists of four valence quarks, irrespective of the tetraquark (molecular) state or twomeson scattering state. Accordingly, in the present case, the Landau singularity only indicates that there exists an intermediate state consists of six valence quarks, irrespective of the $\Sigma_{c} \Xi_{c c}$ two-baryon scattering state or the $\Sigma_{c} \Xi_{c c}$ component in the dibaryon state. The Landau singularity is just a kinematical singularity, not a dynamical singularity $[5,29]$.

If we insist on applying the Landau equation to study the tetraquark molecular states [24,25], we should choose the pole mass to warrant that there exists a pole which corresponds to the mass-shell in pure perturbative calculations. In the case of the $c$-quark, the pole mass $\hat{m}_{c}=$ $1.67 \pm 0.07 \mathrm{GeV}$ from the Particle Data Group [30], $2 \hat{m}_{c}=3.34 \pm 0.14 \mathrm{GeV}>m_{\eta_{c}}$ and $m_{J / \psi}$. It is odd that the charmonium masses lie below the threshold $2 \hat{m}_{c}$ in the QCD sum rules for the $\eta_{c}$ and $J / \psi$.

The nonperturbative contributions play an important role in the QCD sum rules, investigating the perturbative contributions of the order $\mathcal{O}\left(\alpha_{s}^{2}\right)$ alone cannot lead to feasible QCD sum rules for the multiquark states. Furthermore, no feasible QCD sum rules with predictions can be confronted to the experimental data are obtained according to the assertion in Refs. [24,25]. We should bear in mind that the Feynman diagrams at the quark-gluon level involving nonperturbative contributions in the quantum field theory differ greatly from the Feynman diagrams in the intuitive potential quark models. If we insist on understanding the Feynman diagrams intuitively, then the disconnected (or factorizable) Feynman diagrams give masses to the $\Sigma_{c}$ and $\Xi_{c c}$ baryons, the masses are not necessary the physical masses, while the connected (or nonfactorizable) Feynman diagrams contribute attractive interactions to bind the massive $\Sigma_{c}$ and $\Xi_{c c}$ baryons to form molecular states.

In Ref. [31], I refute the assertion of Lucha, Melikhov, and Sazdjian in order and in details, and approve that the
Landau equation is of no use in studying the Feynman diagrams in the QCD sum rules for the tetraquark (molecular) states. The assertion is unreasonable, the tetraquark molecular states begin to receive contributions at the order $\mathcal{O}\left(\alpha_{s}^{0}\right)$ rather than at the order $\mathcal{O}\left(\alpha_{s}^{2}\right)$.

After computing those Feynman diagrams, we obtain the QCD spectral densities through dispersion relation. In this article, we carry out the operator product expansion to the vacuum condensates up to dimension-15, and take into account the vacuum condensates which are vacuum expectations of the quark-gluon operators of the order $\mathcal{O}\left(\alpha_{s}^{k}\right)$ with $k \leq 1$.

There are three light quark propagators and three heavy quark propagators in the correlation functions (9)-(10), if each heavy quark line emits a gluon and each light quark line contributes quark-antiquark pair, we obtain a quark-gluon operator $g_{s} G_{\mu \nu} g_{s} G_{\alpha \beta} g_{s} G_{\lambda \tau} \bar{q} q \bar{q} q \bar{q} q$, which is of dimension 15, and can lead to the vacuum condensates $\left\langle\frac{\alpha_{s} G G}{\pi}\right\rangle\langle\bar{q} q\rangle^{2}\left\langle\bar{q} g_{s} \sigma G q\right\rangle,\left\langle g_{s}^{3} G G G\right\rangle\langle\bar{q} q\rangle^{3}$, and $\left\langle\bar{q} g_{s} \sigma G q\right\rangle^{3}$, we take into account the vacuum condensate $\left\langle\bar{q} g_{s} \sigma G q\right\rangle^{3}$ and neglect the vacuum condensates $\left\langle g_{s}^{3} G G G\right\rangle\langle\bar{q} q\rangle^{3}$ and $\left\langle\frac{\alpha_{s} G G}{\pi}\right\rangle\langle\bar{q} q\rangle^{2}\left\langle\bar{q} g_{s} \sigma G q\right\rangle$. Compared to the $\left\langle\bar{q} g_{s} \sigma G q\right\rangle^{3}$, the $\left\langle g_{s}^{3} G G G\right\rangle\langle\bar{q} q\rangle^{3}$ and $\left\langle\frac{\alpha_{s} G G}{\pi}\right\rangle\langle\bar{q} q\rangle^{2}\left\langle\bar{q} g_{s} \sigma G q\right\rangle$ are neglectful due to the small values. The condensates $\left\langle g_{s}^{3} G G G\right\rangle$, $\left\langle g_{s}^{3} G G G\right\rangle\langle\bar{q} q\rangle$, and $\left\langle\frac{\alpha_{s} G G}{\pi}\right\rangle\left\langle\bar{q} g_{s} \sigma G q\right\rangle$ are not associated with the $\frac{1}{T^{2}}$, and play a tiny role in determining the Borel window, and they are neglected. Furthermore, we neglect the $\left\langle g_{s}^{3} G G G\right\rangle\langle\bar{q} q\rangle^{2}$ due to the small value, it is also beyond the order $\mathcal{O}\left(\alpha_{s}^{1}\right)$.

In summary, we take into account the vacuum condensates $\langle\bar{q} q\rangle,\left\langle\frac{\alpha_{s} G G}{\pi}\right\rangle,\left\langle\bar{q} g_{s} \sigma G q\right\rangle,\langle\bar{q} q\rangle^{2},\langle\bar{q} q\rangle\left\langle\frac{\alpha_{s} G G}{\pi}\right\rangle$, $\langle\bar{q} q\rangle\left\langle\bar{q} g_{s} \sigma G q\right\rangle,\langle\bar{q} q\rangle^{3},\langle\bar{q} q\rangle^{2}\left\langle\frac{\alpha_{s} G G}{\pi}\right\rangle,\left\langle\bar{q} g_{s} \sigma G q\right\rangle^{2},\langle\bar{q} q\rangle^{2} \times$ $\left\langle\bar{q} g_{s} \sigma G q\right\rangle,\langle\bar{q} q\rangle\left\langle\bar{q} g_{s} \sigma G q\right\rangle^{2},\langle\bar{q} q\rangle^{3}\left\langle\frac{\alpha_{s} G G}{\pi}\right\rangle,\left\langle\bar{q} g_{s} \sigma G q\right\rangle^{3}$.

Now we take the quark-hadron duality below the continuum thresholds $s_{0}$ and perform the Borel transformation in regard to $P^{2}=-p^{2}$ to obtain the $\mathrm{QCD}$ sum rules:

$$
\begin{aligned}
& \lambda_{S}^{2} \exp \left(-\frac{M_{S}^{2}}{T^{2}}\right)+\int_{\Delta^{2}}^{s_{0}} d s \rho_{H, S} \exp \left(-\frac{s}{T^{2}}\right)=\int_{9 m_{c}^{2}}^{s_{0}} d s \rho_{S}(s) \exp \left(-\frac{s}{T^{2}}\right), \\
& \lambda_{A}^{2} \exp \left(-\frac{M_{A}^{2}}{T^{2}}\right)+\int_{\Delta^{2}}^{s_{0}} d s \rho_{H, A} \exp \left(-\frac{s}{T^{2}}\right)=\int_{9 m_{c}^{2}}^{s_{0}} d s \rho_{A}(s) \exp \left(-\frac{s}{T^{2}}\right),
\end{aligned}
$$

the very very lengthy expressions of the QCD spectral densities $\rho_{S}(s)$ and $\rho_{A}(s)$ are neglected for simplicity.

We differentiate Eqs. (13) and (14) with respect to $\tau=\frac{1}{T^{2}}$, then eliminate the pole residues $\lambda_{S}$ and $\lambda_{A}$ and obtain the QCD sum rules for the masses of the triply-charmed dibaryon states,

$$
M_{S}^{2}=\frac{-\frac{d}{d \tau}\left[\int_{9 m_{c}^{2}}^{s_{0}} d s \rho_{S}(s) \exp (-s \tau)-\int_{\Delta^{2}}^{s_{0}} d s \rho_{H, S}(s) \exp (-s \tau)\right]}{\int_{9 m_{c}^{2}}^{s_{0}} d s \rho_{S}(s) \exp (-s \tau)-\int_{\Delta^{2}}^{s_{0}} d s \rho_{H, S}(s) \exp (-s \tau)},
$$




$$
M_{A}^{2}=\frac{-\frac{d}{d \tau}\left[\int_{9 m_{c}^{2}}^{s_{0}} d s \rho_{A}(s) \exp (-s \tau)-\int_{\Delta^{2}}^{s_{0}} d s \rho_{H, A}(s) \exp (-s \tau)\right]}{\int_{9 m_{c}^{2}}^{s_{0}} d s \rho_{A}(s) \exp (-s \tau)-\int_{\Delta^{2}}^{s_{0}} d s \rho_{H, A}(s) \exp (-s \tau)}
$$

If the QCD sum rules can be saturated with the scalar and axialvector dibaryon states alone, we set $\rho_{H, S}=\rho_{H, A}=0$ in Eqs. (13)-(16), we obtain the two sets of QCD sum rules for the dibaryon states, and refer those QCD sum rules as the QCDSR I, and refer the QCD sum rules in Eqs. (13)(16) as the QCDSR II. On the other hand, if the QCD sum rules can be saturated with the scalar and axialvector twobaryon scattering states alone, we set $\lambda_{S}=\lambda_{A}=0$, we obtain the two QCD sum rules,

$$
\begin{aligned}
& \int_{\Delta^{2}}^{s_{0}} d s \rho_{H, S} \exp \left(-\frac{s}{T^{2}}\right)=\kappa_{S} \int_{9 m_{c}^{2}}^{s_{0}} d s \rho_{S}(s) \exp \left(-\frac{s}{T^{2}}\right), \\
& \int_{\Delta^{2}}^{s_{0}} d s \rho_{H, A} \exp \left(-\frac{s}{T^{2}}\right)=\kappa_{A} \int_{9 m_{c}^{2}}^{s_{0}} d s \rho_{A}(s) \exp \left(-\frac{s}{T^{2}}\right),
\end{aligned}
$$

where we introduce the parameters $\kappa_{S}$ and $\kappa_{A}$ to parametrize the deviations from the value 1 . Thereafter, we refer the QCD sum rules in Eqs. (17) and (18) as the QCDSR III.

\section{NUMERICAL RESULTS AND DISCUSSIONS}

We choose the standard values of the vacuum condensates $\langle\bar{q} q\rangle=-(0.24 \pm 0.01 \mathrm{GeV})^{3},\left\langle\bar{q} g_{s} \sigma G q\right\rangle=m_{0}^{2}\langle\bar{q} q\rangle$, $m_{0}^{2}=(0.8 \pm 0.1) \mathrm{GeV}^{2},\left\langle\frac{\alpha_{s} G G}{\pi}\right\rangle=(0.33 \mathrm{GeV})^{4}$ at the energy scale $\mu=1 \mathrm{GeV}[27,32,33]$, and choose the $\overline{\mathrm{MS}}$ mass $m_{c}\left(m_{c}\right)=(1.275 \pm 0.025) \mathrm{GeV}$ from the Particle Data Group [30], and set $m_{u}=m_{d}=0$. We take into account the energy-scale dependence of the input parameters,

$$
\begin{aligned}
\langle\bar{q} q\rangle(\mu) & =\langle\bar{q} q\rangle(1 \mathrm{GeV})\left[\frac{\alpha_{s}(1 \mathrm{GeV})}{\alpha_{s}(\mu)}\right]^{\frac{12}{25}}, \\
\left\langle\bar{q} g_{s} \sigma G q\right\rangle(\mu) & =\left\langle\bar{q} g_{s} \sigma G q\right\rangle(1 \mathrm{GeV})\left[\frac{\alpha_{s}(1 \mathrm{GeV})}{\alpha_{s}(\mu)}\right]^{\frac{2}{25}}, \\
m_{c}(\mu) & =m_{c}\left(m_{c}\right)\left[\frac{\alpha_{s}(\mu)}{\alpha_{s}\left(m_{c}\right)}\right]^{\frac{12}{25}}, \\
\alpha_{s}(\mu)= & \frac{1}{b_{0} t}\left[1-\frac{b_{1}}{b_{0}^{2}} \frac{\log t}{t}\right. \\
& \left.+\frac{b_{1}^{2}\left(\log ^{2} t-\log t-1\right)+b_{0} b_{2}}{b_{0}^{4} t^{2}}\right],
\end{aligned}
$$

where $t=\log \frac{\mu^{2}}{\Lambda^{2}}, \quad b_{0}=\frac{33-2 n_{f}}{12 \pi}, \quad b_{1}=\frac{153-19 n_{f}}{24 \pi^{2}}, \quad b_{2}=$ $\frac{2857-\frac{5033}{9} n_{f}+\frac{325}{27} n_{f}^{2}}{128 \pi^{3}}, \Lambda=210 \mathrm{MeV}, 292 \mathrm{MeV}$, and $332 \mathrm{MeV}$ for the flavors $n_{f}=5,4$, and 3, respectively [30,34,35], and evolve all the input parameters to the best energy scales to extract the dibaryon masses with the flavor $n_{f}=4$.

In the article, we study the baryon-baryon type six-quark states (dibaryon states) or hexaquark states, which have three charmed quarks. Those six-quark systems are characterized by the effective charmed quark mass or constituent quark mass $\mathbb{M}_{c}$ and the virtuality $V=\sqrt{M_{D}^{2}-\left(3 \mathbb{M}_{c}\right)^{2}}$, where the $D$ denotes the dibaryon states. We set the energy scales of the QCD spectral densities to be $\mu=V$, it is a straight forward extension of the energy scale formula $\mu=\sqrt{M_{X / Y / Z}^{2}-\left(2 \mathbb{M}_{c}\right)^{2}}$ suggested for the hidden-charm tetraquark molecular states to the triply charmed dibaryon states [19]. In this article, we choose the updated value $\mathbb{M}_{c}=1.85 \mathrm{GeV}$ [20], and take the energy scale formula,

$$
\mu=\sqrt{M_{D}^{2}-\left(3 M_{c}\right)^{2}}
$$

as a powerful constraint to satisfy.

The relevant baryon masses and pole residues are $m_{\Sigma_{c}^{++}}=2453.97 \mathrm{MeV}, \quad m_{\Sigma_{c}^{+}}=2452.9 \mathrm{MeV}, \quad m_{\Sigma_{c}^{0}}=$ $2453.75 \mathrm{MeV}, m_{\Xi_{c c}^{++}}=3621.2 \mathrm{MeV}$ from the Particle Data Group [30], $m_{\Sigma}=2.40 \mathrm{GeV}, m_{\Xi}=3.63 \mathrm{GeV}, \lambda_{\Sigma}=$ $0.045 \mathrm{GeV}^{3}$ and $\lambda_{\Xi}=0.102 \mathrm{GeV}^{3}$ from the QCD sum rules [36,37]. In this article, we choose the values $m_{\Sigma}=$ $2.45 \mathrm{GeV}$ and $m_{\Xi}=3.62 \mathrm{GeV}$. We vary the baryon masses $m_{\Sigma}$ and $m_{\Xi}$ slightly, which leads to neglectful changes. In this article, we take the continuum threshold parameter as $\sqrt{s_{0}}=m_{\Sigma}+m_{\Xi}+(0.5 \sim 0.7) \mathrm{GeV}=6.1 \pm$ $0.6 \mathrm{GeV}$ to take into account the contributions from the $\Sigma_{c}$ and $\Xi_{c c}$ baryon states sufficiently.

We define the pole contributions PC as

$$
\mathrm{PC}=\frac{\int_{9 m_{c}^{2}}^{s_{0}} d s \rho_{S / A}(s) \exp \left(-\frac{s}{T^{2}}\right)}{\int_{9 m_{c}^{2}}^{\infty} d s \rho_{S / A}(s) \exp \left(-\frac{s}{T^{2}}\right)} .
$$

For the multiquark states, it is difficult to satisfy the pole dominance criterion. The energy scale formula, see Eq. (20), can enhance the pole contributions significantly, and also can improve the convergent behaviors of the operator product expansion substantially. If the operator product expansion is convergent, the contributions of the higher dimensional vacuum condensates $D(n)$ with $n \geq 10$ play a minor important role,

$$
D(n)=\frac{\int_{9 m_{c}^{2}}^{s_{0}} d s \rho_{S / A ; n}(s) \exp \left(-\frac{s}{T^{2}}\right)}{\int_{9 m_{c}^{2}}^{s_{0}} d s \rho_{S / A}(s) \exp \left(-\frac{s}{T^{2}}\right)}
$$


TABLE I. The Borel parameters, continuum threshold parameters, energy scales, pole contributions, masses and pole residues for the triply charmed dibaryon states from the QCD sum rules I and II.

\begin{tabular}{lcccccc}
\hline \hline$J^{P}$ & $T^{2}\left(\mathrm{GeV}^{2}\right)$ & $\sqrt{s_{0}}(\mathrm{GeV})$ & $\mu(\mathrm{GeV})$ & Pole & $M(\mathrm{GeV})$ & $\lambda\left(\mathrm{GeV}^{8}\right)$ \\
\hline $0^{+}(\mathrm{I})$ & $3.9-4.5$ & $6.7 \pm 0.1$ & 2.4 & $(38-63) \%$ & $6.05 \pm 0.13$ & $(1.45 \pm 0.30) \times 10^{-2}$ \\
$0^{+}(\mathrm{II})$ & & & & & $6.04 \pm 0.13$ & $(1.39 \pm 0.30) \times 10^{-2}$ \\
$1^{+}(\mathrm{I})$ & $4.1-4.7$ & $6.7 \pm 0.1$ & 2.4 & $(38-62) \%$ & $6.03 \pm 0.13$ & $(1.44 \pm 0.29) \times 10^{-2}$ \\
$1^{+}(\mathrm{II})$ & & & & & $6.02 \pm 0.13$ & $(1.39 \pm 0.28) \times 10^{-2}$ \\
\hline \hline
\end{tabular}

where the subscript $n$ in the QCD spectral densities $\rho_{S / A ; n}(s)$ denotes the vacuum condensates of dimension $n$.

We obtain the Borel windows and the best energy scales of the QCD spectral densities for the QCDSR I, which are shown in Table I, via trial and error. Now it is straight forward to get the pole contributions, the pole contributions are as large as (38-63)\%, it is large enough to extract the dibaryon masses. In the QCD sum rules for the multiquark

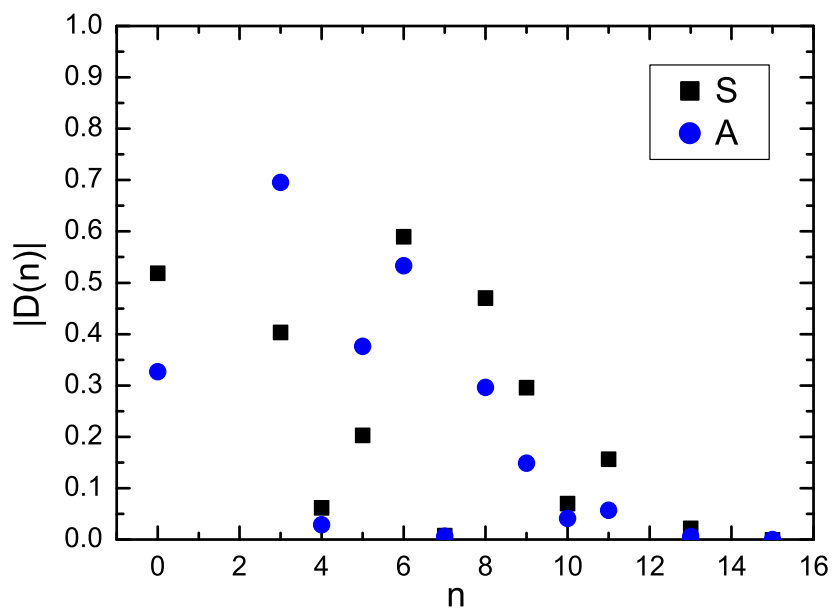

FIG. 3. The absolute values of the contributions of the vacuum condensates of dimension $n$ for central values of the input parameters in the QCDSR I/II, where the $S$ and $A$ represent the scalar and axialvector dibaryon states respectively.

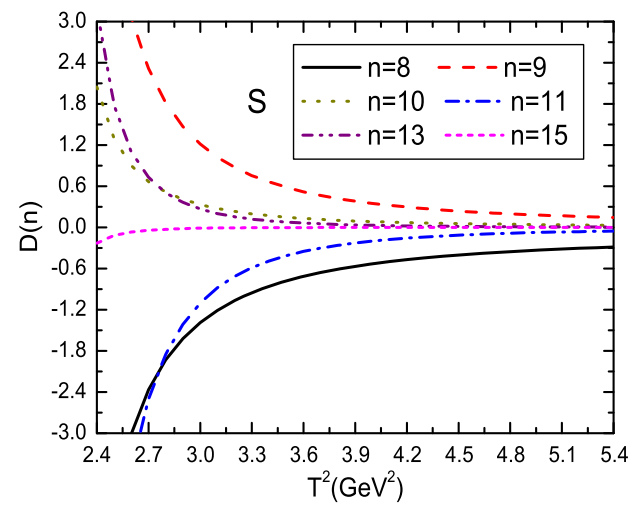

states, the pole contributions are usually small due to the QCD spectral densities $\rho_{\mathrm{QCD}}(s) \propto s^{n}$ with the largest value $n \geq 4$ in the zero quark mass limit [38].

In Fig. 3, we plot the absolute values of the $D(n)$ for the central values of the input parameters. Although the perturbative contributions are not large enough to dominate the QCD sum rules, the vacuum condensate $\langle\bar{q} q\rangle^{2}$ with the dimension 6 serves as a milestone, the absolute values of the contributions of the vacuum condensates with $n \geq 6$ decrease monotonically and quickly with increase of the dimension except for the vacuum condensate $\langle\bar{q} q\rangle\left\langle\frac{\alpha_{s} G G}{\pi}\right\rangle$, which plays a tiny role. The convergent behavior of the operator product expansion is very good.

Although the higher dimensional vacuum condensates play a minor important role in the Borel windows, they play an important role in determining the Borel windows, we should take them into account in a consistent way. In Fig. 4, we plot the values of the $D(n)$ for $n \geq 8$ with variations of the Borel parameters for the central values of the other input parameters. From Fig. 4, we observe that the contributions of the vacuum condensates of dimensions $8,9,10,11$, and 13 are large at the region $T^{2}<3.9 \mathrm{GeV}^{2}$, we should choose $T^{2} \geq 3.9 \mathrm{GeV}^{2}$, while the contributions of the vacuum condensates of dimension 15 are very small in the whole regions. In fact, the vacuum condensate $\left\langle\bar{q} g_{s} \sigma G q\right\rangle^{3}$ is the vacuum expectation of the operator of the order $\mathcal{O}\left(\alpha_{s}^{\frac{3}{2}}\right)$, which is beyond the truncation $\mathcal{O}\left(\alpha_{s}^{k}\right)$ with $k \leq 1$.

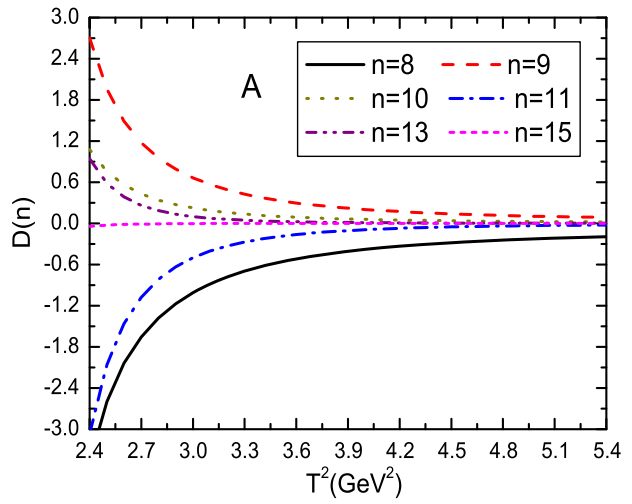

FIG. 4. The contributions of the higher dimensional vacuum condensates with variations of the Borel parameters $T^{2}$ in the QCDSR I/ II, where the $S$ and $A$ represent the scalar and axialvector dibaryon states respectively. 

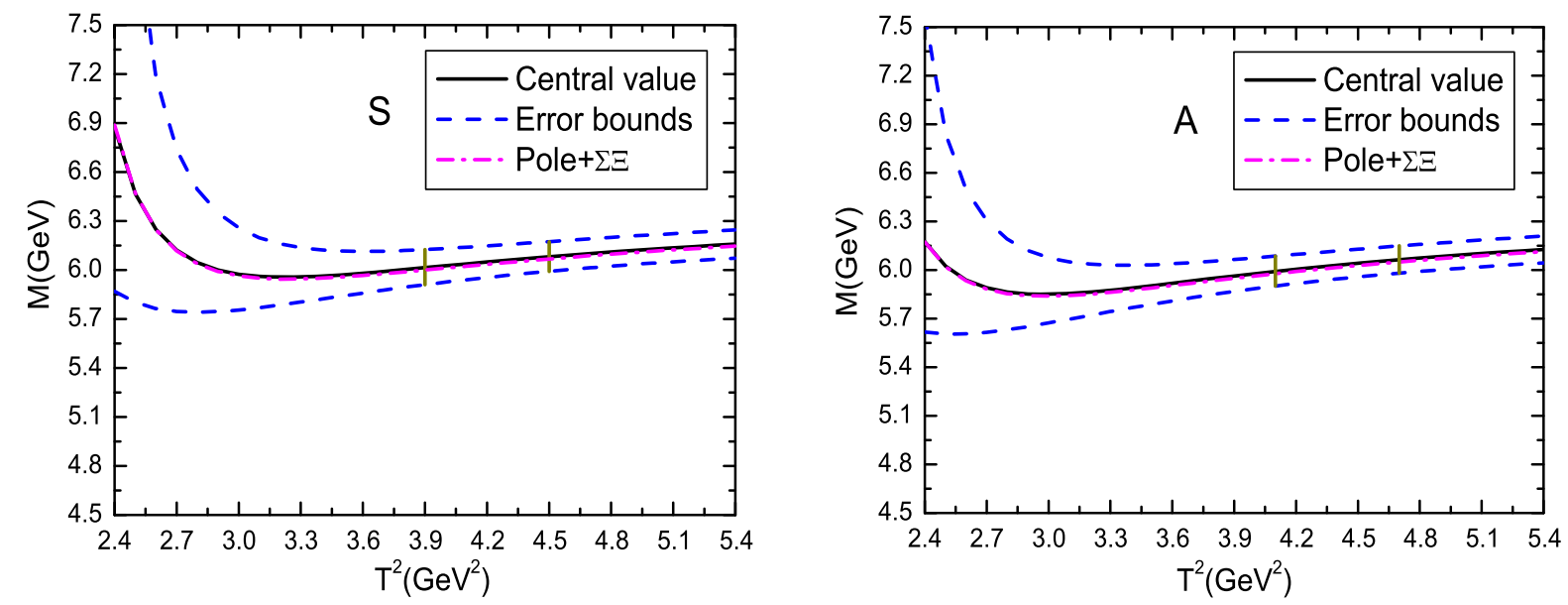

FIG. 5. The masses of the dibaryon states with variations of the Borel parameters $T^{2}$ from the QCDSR I, where the $S$ and $A$ represent the scalar and axialvector dibaryon states respectively, the Pole $+\Sigma \Xi$ corresponds to the results from the QCDSR II.

Now we estimate the contributions of the vacuum condensates $\langle\bar{q} q\rangle^{3}\left\langle g_{s}^{3} G G G\right\rangle$, which are also of dimension 15 . If each light quark propagator contributes a quark-antiquark pair, which forms the quark condensate, $S_{i j}(x) \rightarrow$ $-\frac{1}{12}\langle\bar{q} q\rangle \delta_{i j}$, we can write down the corresponding contribution $\Pi_{S}(p)$ (the corresponding contribution $\Pi_{\mu \nu}(p)$ can be treated similarly),

$$
\Pi_{S}(p)=-\frac{2}{27}\langle\bar{q} q\rangle^{3} \Pi_{c c c}(p)+\cdots,
$$

where

$$
\begin{aligned}
\Pi_{c c c}(p)= & i \varepsilon^{l m n} \varepsilon^{l^{\prime} m^{\prime} n} \int d^{4} x e^{i p \cdot x} \operatorname{Tr}\left[\gamma_{\beta} S_{c}^{m m^{\prime}}(x) \gamma^{\beta} C S_{c}^{l l^{\prime} T}(x) C\right] \\
& \times \operatorname{Tr}\left[C S_{c}^{k k T}(x) C\right] .
\end{aligned}
$$

The correlation function $\Pi_{c c c}(p)$ has three heavy quark propagators, just like in the QCD sum rules for the triply heavy baryon states [39]. The vacuum condensates in the correlation functions $\Pi_{S}(p)$ and $\Pi_{c c c}(p)$ have the following relations,

$$
\begin{aligned}
\langle\bar{q} q\rangle^{3} & \leftrightarrow \text { perturbative term, } \\
\langle\bar{q} q\rangle^{3}\left\langle\frac{\alpha_{s} G G}{\pi}\right\rangle & \leftrightarrow\left\langle\frac{\alpha_{s} G G}{\pi}\right\rangle, \\
\langle\bar{q} q\rangle^{3}\left\langle g_{s}^{3} G G G\right\rangle & \leftrightarrow\left\langle g_{s}^{3} G G G\right\rangle .
\end{aligned}
$$

In the QCD sum rules for the triply heavy baryon states, we usually take into account the perturbative terms and the gluon condensates $\left\langle\frac{\alpha_{S} G G}{\pi}\right\rangle$, and neglect the contributions of the three-gluon condensates due to their small values [39]. We expect that the contributions of the three-gluon condensates in the $\Pi_{c c c}(p)$ can be neglected, just like in the case of the triply heavy baryon states, therefore, the corresponding vacuum condensates $\langle\bar{q} q\rangle^{3}\left\langle g_{s}^{3} G G G\right\rangle$ in the $\Pi_{S}(p)$ can also be neglected.

If we want to calculate the three-gluon condensates, we should take into account the three-gluon operators neglected in the full propagators in Eqs. (11) and (12). In Ref. [40], we calculate the contributions of the vacuum condensates up to dimension- 6 in the operator product expansion, study the masses and decay constants of the heavy tensor mesons with the QCD sum rules. In calculations, we observe that the contributions come from the gluon condensates and three-gluon condensates are about $10 \%$ and $0.2 \%$ respectively for the charmed mesons. It is indeed that the contributions of the three-gluon condensates are much smaller than that of the gluon condensates.

From Fig. 3, we can see that the contributions of the vacuum condensates $\langle\bar{q} q\rangle\left\langle\bar{q} g_{s} \sigma G q\right\rangle^{2}$ and $\langle\bar{q} q\rangle^{3}\left\langle\frac{\alpha_{s} G G}{\pi}\right\rangle$, which are of dimension 13 and have two gluon operators, are tiny. We can draw the conclusion tentatively that the contributions of the vacuum condensates $\left\langle\bar{q} g_{s} \sigma G q\right\rangle^{3}$, $\left\langle\frac{\alpha_{s} G G}{\pi}\right\rangle\langle\bar{q} q\rangle^{2}\left\langle\bar{q} g_{s} \sigma G q\right\rangle$ and $\langle\bar{q} q\rangle^{3}\left\langle g_{s}^{3} G G G\right\rangle$, which are of dimension 15 and have three gluon operators, are even tiny. It is indeed that the contributions of the vacuum condensates $\left\langle\bar{q} g_{s} \sigma G q\right\rangle^{3}$, which are of dimension 15 , are even tiny, see Figs. 3 and 4 . The higher dimensional vacuum condensates play a minor important role in the Borel windows, the operator product expansion is well convergent.

Now we take into account all uncertainties of the input parameters, and obtain the values of the masses and pole residues of the triply charmed dibaryon states from the QCDSR I and II respectively, which are shown explicitly in Table I and Figs. 5 and 6, where the regions between the two vertical lines are the Borel windows. In Figs. 5 and 6, we plot the masses and pole residues of the triply-charmed dibaryon states in much larger ranges than the Borel windows. Flat platforms appear at the Borel windows both for the masses and pole residues, it is reliable to extract the 

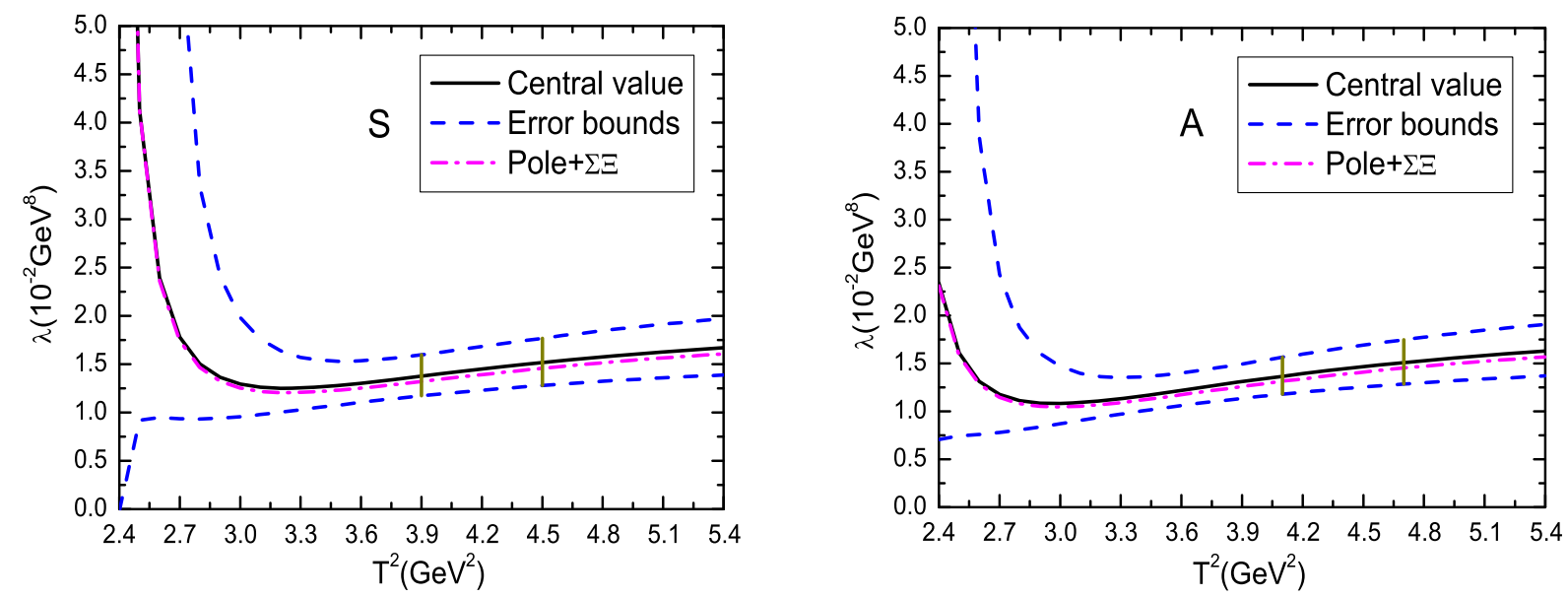

FIG. 6. The pole residues of the dibaryon states with variations of the Borel parameters $T^{2}$ from the QCDSR I, where the $S$ and $A$ represent the scalar and axialvector dibaryon states respectively, the Pole $+\Sigma \Xi$ corresponds to the results from the QCDSR II.

dibaryon masses. From Table I, we can see that the central values of the dibaryon masses from the QCDSR I satisfy the energy scale formula $\mu=\sqrt{M_{D}^{2}-\left(3 \mathrm{M}_{c}\right)^{2}}$. If we take into account the two-baryon scattering states, we obtain a slightly smaller masses and pole residues for the dibaryon states from the QCDSR II, see Table I and Figs. 5 and 6. The effects of the two baryon scattering states are rather small and can be neglected safely.

In Ref. [11], the lattice QCD calculations indicate that the color-singlet-color-singlet type currents with $J^{P}=1^{+}$ have nonvanishing couplings with the heavy dibaryon states, which lie below the corresponding two-baryon thresholds. In Ref. [9], the calculations based on the one-boson-exchange model indicate that there exist attractive interactions between the $\Xi_{c c}$ and $\Sigma_{c}$ in the channels $I\left(J^{P}\right)=\frac{1}{2}\left(0^{+}\right), \frac{1}{2}\left(1^{+}\right)$, and $\frac{3}{2}\left(0^{+}\right)$. In Ref. [8], the heavy antiquark-diquark symmetry is used to study the mass-splitting between the $J^{P}=0^{+}$and $1^{+}$

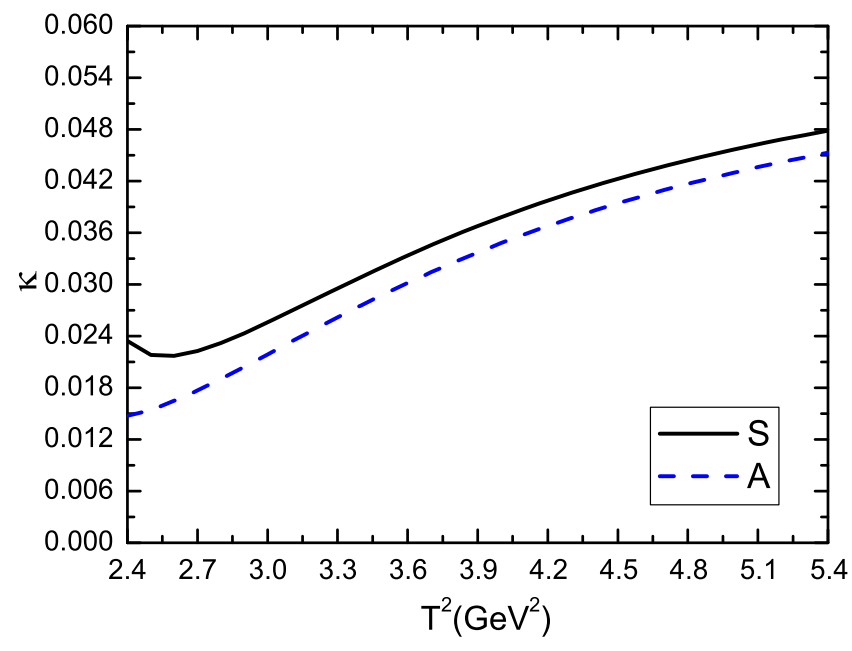

FIG. 7. The parameters $\kappa$ with variations of the Borel parameters $T^{2}$ from the QCDSR III, where the $S$ and $A$ represent the scalar and axialvector dibaryon states respectively.
$\Xi_{c c} \Sigma_{c}$ dibaryon states in a model-independent way. In the present work, the predictions of the central values $M_{S}=$ $6.05 \mathrm{GeV}(6.04 \mathrm{GeV})$ and $M_{A}=6.03 \mathrm{GeV}(6.02 \mathrm{GeV})$ from the QCDSR I (QCDSR II) are consistent with the previous works $[8,9,11]$.

In Fig. 7, we plot the parameters $\kappa_{S}$ and $\kappa_{A}$ with variations of the Borel parameters for the central values of the input parameters shown in Table I. From the figure, we can see that the values $\kappa_{S} \ll 1$ and $\kappa_{A} \ll 1$, furthermore, the values of the $\kappa_{S}$ and $\kappa_{A}$ increase monotonically and quickly with increase of the Borel parameters, no flat platforms can be obtained. If we choose smaller energy scale, say $\mu=1 \mathrm{GeV}$ (which is chosen in the QCD sum rules for the $\Sigma_{c}$ and $\left.\Xi_{c c}[36,37]\right)$, we can obtain very large values for the $\kappa_{S}$ and $\kappa_{A}$ with poor pole contributions, the values of the $\kappa_{S}$ and $\kappa_{A}$ decrease monotonically and quickly with increase of the Borel parameters, on the other hand, the convergent behaviors of the operator product expansion are very bad. We can draw the conclusion tentatively that the two-baryon scattering states cannot saturate the QCD sum rules and play a minor important role and can be neglected, which approves the observation obtained in Sec. II, the molecular states begin to receive contributions at the order $\mathcal{O}\left(\alpha_{s}^{0}\right)$, not at the order $\mathcal{O}\left(\alpha_{s}^{2}\right)$.

\section{CONCLUSION}

In this article, we construct the color-singlet-colorsinglet type currents to interpolate the scalar and axialvector $\Xi_{c c} \Sigma_{c}$ dibaryon states, and study them with QCD sum rules in details by carrying out the operator product expansion up to the vacuum condensates of dimension 15. At the hadron side, we take into account both the contributions of the dibaryon states and two-baryon scattering sates, and explore the existence or nonexistence of the $\Xi_{c c} \Sigma_{c}$ dibaryon states in three cases. As there exist three valance $c$-quarks, the QCD sum rules are sensitive to the $c$-quark mass or the energy scale of the QCD spectral 
densities, we determine the best energy scales with the energy scale formula $\mu=\sqrt{M_{D}^{2}-\left(3 \mathbb{M}_{c}\right)^{2}}$. The numerical results indicate that the two-baryon scattering states cannot saturate the QCD sum rules and play a minor important role, the dominant contributions come from the dibaryon states. Or it is necessary to introduce the dibaryon states to embody the net effects. The color-singlet-color-singlet type currents couple potentially to the molecular states, not to the two-particle scattering states. In the operator product expansion, the molecular states begin to receive contributions at the order $\mathcal{O}\left(\alpha_{s}^{0}\right)$, not at the order $\mathcal{O}\left(\alpha_{s}^{2}\right)$. We can search for the triply charmed dibaryon states at the $\mathrm{LHCb}$, Belle II, CEPC, FCC, ILC in the future.

\section{ACKNOWLEDGMENTS}

This work is supported by National Natural Science Foundation, Grant No. 11775079.
[1] P. Adlarson et al., Phys. Rev. C 90, 035204 (2014).

[2] P. Adlarson et al., Phys. Rev. Lett. 106, 242302 (2011); 112, 202301 (2014).

[3] H. Clement, Prog. Part. Nucl. Phys. 93, 195 (2017).

[4] S. K. Choi et al., Phys. Rev. Lett. 91, 262001 (2003).

[5] F. K. Guo, C. Hanhart, U. G. Meissner, Q. Wang, Q. Zhao, and B. S. Zou, Rev. Mod. Phys. 90, 015004 (2018).

[6] R. Aaij et al., Phys. Rev. Lett. 115, 072001 (2015).

[7] R. Aaij et al., Phys. Rev. Lett. 122, 222001 (2019).

[8] Y. W. Pan, M. Z. Liu, F. Z. Peng, M. S. Sanchez, L. S. Geng, and M. P. Valderrama, Phys. Rev. D 102, 011504 (2020).

[9] R. Chen, F. L. Wang, A. Hosaka, and X. Liu, Phys. Rev. D 97, 114011 (2018).

[10] W. Meguro, Y. R. Liu, and M. Oka, Phys. Lett. B 704, 547 (2011); L. Meng, N. Li, and S. L. Zhu, Phys. Rev. D 95, 114019 (2017).

[11] P. Junnarkar and N. Mathur, Phys. Rev. Lett. 123, 162003 (2019).

[12] H. Huang, J. Ping, and F. Wang, Phys. Rev. C 89, 035201 (2014); J. Vijande, A. Valcarce, J. M. Richard, and P. Sorba, Phys. Rev. D 94, 034038 (2016).

[13] R. Aaij et al., Phys. Rev. Lett. 119, 112001 (2017).

[14] N. Kodama, M. Oka, and T. Hatsuda, Nucl. Phys. A580, 445 (1994).

[15] H. X. Chen, E. L. Cui, W. Chen, T. G. Steele, and S. L. Zhu, Phys. Rev. C 91, 025204 (2015).

[16] R. D. Matheus, S. Narison, M. Nielsen, and J. M. Richard, Phys. Rev. D 75, 014005 (2007); Z. G. Wang, Eur. Phys. J. C 63, 115 (2009); W. Chen and S. L. Zhu, Phys. Rev. D 83, 034010 (2011); J. R. Zhang, M. Zhong, and M. Q. Huang, Phys. Lett. B 704, 312 (2011); J. R. Zhang, Phys. Rev. D 87, 116004 (2013); C. F. Qiao and L. Tang, Eur. Phys. J. C 74, 2810 (2014); Z. G. Wang, Commun. Theor. Phys. 63, 466 (2015); Z. G. Wang and Y. F. Tian, Int. J. Mod. Phys. A 30, 1550004 (2015); Z. G. Wang, Eur. Phys. J. C 79, 29 (2019).

[17] Z. G. Wang and T. Huang, Phys. Rev. D 89, 054019 (2014).

[18] F. S. Navarra and M. Nielsen, Phys. Lett. B 639, 272 (2006); J. M. Dias, F. S. Navarra, M. Nielsen, and C. M. Zanetti, Phys. Rev. D 88, 016004 (2013); W. Chen, T. G. Steele, H. X. Chen, and S. L. Zhu, Eur. Phys. J. C 75, 358 (2015); Z. G. Wang and T. Huang, Nucl. Phys. A930, 63 (2014);
S. S. Agaev, K. Azizi, and H. Sundu, Phys. Rev. D 93, 074002 (2016); Z. G. Wang and J. X. Zhang, Eur. Phys. J. C 78, 14 (2018); Z. G. Wang, Int. J. Mod. Phys. A 34, 1950110 (2019).

[19] Z. G. Wang and T. Huang, Eur. Phys. J. C 74, 2891 (2014); Z. G. Wang, Eur. Phys. J. C 74, 2963 (2014).

[20] Z. G. Wang, Chin. Phys. C 41, 083103 (2017).

[21] H. X. Chen, W. Chen, X. Liu, T. G. Steele, and S. L. Zhu, Phys. Rev. Lett. 115, 172001 (2015); K. Azizi, Y. Sarac, and H. Sundu, Phys. Rev. D 95, 094016 (2017).

[22] Z. G. Wang, Int. J. Mod. Phys. A 34, 1950097 (2019).

[23] Z. G. Wang, Eur. Phys. J. C 76, 70 (2016); Z. G. Wang and T. Huang, Eur. Phys. J. C 76, 43 (2016).

[24] W. Lucha, D. Melikhov, and H. Sazdjian, Phys. Rev. D 100, 014010 (2019); 100, 074029 (2019).

[25] W. Lucha, D. Melikhov, and H. Sazdjian, Eur. Phys. J. C 77, 866 (2017).

[26] L. D. Landau, Nucl. Phys. 13, 181 (1959).

[27] L. J. Reinders, H. Rubinstein, and S. Yazaki, Phys. Rep. 127, 1 (1985).

[28] P. Pascual and R. Tarrach, QCD: Renormalization for the Practitioner (Springer Berlin Heidelberg, 1984).

[29] F. K. Guo, Proc. Sci., Hadron 2017 (2018) 015; F. K. Guo, X. H. Liu, and S. Sakai, Prog. Part. Nucl. Phys. 112, 103757 (2020).

[30] C. Patrignani et al., Chin. Phys. C 40, 100001 (2016).

[31] Z. G. Wang, Phys. Rev. D 101, 074011 (2020).

[32] M. A. Shifman, A. I. Vainshtein, and V. I. Zakharov, Nucl. Phys. B147, 385 (1979); B147, 448 (1979).

[33] P. Colangelo and A. Khodjamirian, arXiv:hep-ph/0010175.

[34] S. Narison and R. Tarrach, Phys. Lett. 125B, 217 (1983).

[35] S. Narison, Cambridge Monogr. Part. Phys., Nucl. Phys., Cosmol. 17, 1 (2007).

[36] Z. G. Wang, Phys. Lett. B 685, 59 (2010).

[37] Z. G. Wang, Eur. Phys. J. C 78, 826 (2018).

[38] Z. G. Wang, Nucl. Phys. A791, 106 (2007).

[39] J. R. Zhang and M. Q. Huang, Phys. Lett. B 674, 28 (2009); Z. G. Wang, Commun. Theor. Phys. 58, 723 (2012); T. M. Aliev, K. Azizi, and M. Savci, J. Phys. G 41, 065003 (2014).

[40] Z. G. Wang and Z. Y. Di, Eur. Phys. J. A 50, 143 (2014). 